\title{
Investigation of the capability of the Compact Polarimetry mode to Reconstruct Full Polarimetry mode using RADARSAT2 data
}

\author{
Boularbah Souissi $^{1}$, Mounira Ouarzeddine ${ }^{2}$, Aichouche Belhadj-Aissa ${ }^{3}$ \\ ${ }^{1}$ Infotronic, Department of Physics, Faculty of Science, UMBB University, Boumerdes, Algeria \\ ${ }^{3,2}$ Faculty of Electronics and Computational Science, USTHB University, Algiers, Algeria \\ *corresponding author, E-mail: m. ouarzeddine@yahoo.fr
}

\begin{abstract}
Recently, there has been growing interest in dual-pol systems that transmit one polarization and receive two polarizations. Souyris et al. proposed a DP mode called compact polarimetry (CP) which is able to reduce the complexity, cost, mass, and data rate of a SAR system while attempting to maintain many capabilities of a fully polarimetric system. This paper provides a comparison of the information content of full quad-pol data and the pseudo quad-pol data derived from compact polarimetric SAR modes. A pseudo-covariance matrix can be reconstructed following Souyris's approach and is shown to be similar to the full polarimetric (FP) covariance matrix. Both the polarimetric signatures based on the kennaugh matrix and the Freeman and Durden decomposition in the context of this compact polarimetry mode are explored. The Freeman and Durden decomposition is used in our study because of its direct relationship to the reflection symmetry.

We illustrate our results by using the polarimetric SAR images of Algiers city in Algeria acquired by the RadarSAT2 in C-band.
\end{abstract}

\section{Introduction}

The polarimetric radar synthetic aperture radar (PolSAR) images are formed by radar echoes of various combinations of transmitting and receiving polarizations from scattering media [1]. Single polarimetric radar systems (SP) operate with single fixed polarization antenna for both electromagnetic wave transmission and reception, for example transmitting horizontally polarized radiation and receiving horizontal polarization.

The full polarimetric SAR (FP-PolSAR) design architectures are built around the standard linear basis, i.e., horizontal $(\mathrm{H})$ and vertical $(\mathrm{V})$. On transmission, the radar interleaves pulses with $\mathrm{H}$ and $\mathrm{V}$ polarizations. On-receive, both polarizations are simultaneously and coherently recorded [1,2]. This quad-pol system produces the scattering matrix containing the four backscatter measurements $(\mathrm{HH}, \mathrm{HV}, \mathrm{VH}$ and $\mathrm{VV})$ which allow much more information to be extracted from target scene. Indeed, FP mode has proven its increased potential compared to a single channel acquisition, but suffer from an increase in the pulse repetition frequency by a factor of two and an increase in the data rate by a factor of four over single polarization [3]. Their imaged swaths also are halved, resulting in reduced coverage and a degraded revisiting time [3]. The L-band PALSAR on board of the Japanese ALOS, for example, can only image $35-\mathrm{km}$ swaths at incidence angles of less than $27^{\circ}$ when operating in FP mode. Similarly, the Canadian Radarsat-2 offers a quadpolarimetric mode over a wider range of incidence angles $\left(20^{\circ}-40^{\circ}\right)$ but a swath width of only $25 \mathrm{~km}$ [4].

Recently, dual-mode partially polarimetric SAR systems (DP) have been proposed such us Envisat ASAR, the Japanese L-band PalSAR, the European X-band TerraSAR$\mathrm{X}$, and the Canadian C-band RadarSAT 2, where one polarization $(\mathrm{H}$ or $\mathrm{V})$ is transmitted, whereas two are received. These DPmodes collect only half of the full scattering matrix, either $(\mathrm{HH}, \mathrm{VH})$ or $(\mathrm{VV}, \mathrm{HV})$. This reduces both the data processing requirements and the information content of the polarimetric imagery. The acquired data set has the same coverage as for the single transmit polarization, but provides added information due to the two independent channels on-receive. From a mission standpoint, the DP imaging modes collect a wider swath width, and hence greater area coverage, as compared to quad-pol (FP) imagery. However, the per-pixel information content is less for DP imagery than for quad-pol imagery, which tends to favor quad-pol data collection [5].

Other system configurations have been also proposed and called compact polarimetry [6,7]. In these polarimetric configurations, only one transmit/receive cycle is required instead of two in a quad-pol system, reducing the pulse repetition frequency and data rates by a factor of two for a given swath width. Souyris et al. [6] introduced the $\pi / 4$ compact polarimetric mode, in which the transmitted polarization is the superposition of linear horizontal and vertical polarizations $\mathrm{H}+\mathrm{V}$, resulting in a linear polarization oriented at $45^{\circ}$ with respect to the horizontal. The radar receives returns in horizontal and vertical polarizations. Another hybrid DP mode is the circular transmit, linear receive (CTLR) mode [7]. As the name suggests either a left or right circularly-polarized signal is transmitted and both $\mathrm{H}$ and $\mathrm{V}$ polarizations are coherently received. The polarimetric models employed assume both reflection symmetric scattering, and a relationship between the linear coherence and the cross-polarization ratio to construct a full reflection symmetric polarization matrix from the $2 \times 2$ covariance data [6]. An equivalent covariance or coherency 
matrix may be reconstructed to produce the so-called pseudo quad-pol data that accurately reproduces the full quad-pol data.

The compact polarimetry was proposed to assess various architecture designs that could be implemented on low-cost/low-mass. In that context, the comparison between FP versus DP is a subject of most importance.

This paper provides a comparison of the information content of full quad-pol data and the pseudo quad-pol data derived from compact polarimetric SAR modes. A pseudocovariance matrix can be reconstructed following Souyris's approach and is shown to be similar to the full polarimetric (FP) covariance matrix. Both the polarimetric signatures based on the kennaugh matrix and the Freeman and Durden decomposition in the context of this compact polarimetry mode are explored. The Freeman and Durden decomposition is used in our study because of its direct relationship to the reflection symmetry.

We illustrate our results by using the polarimetric SAR images of Algiers city in Algeria acquired by the RadarSAT2 in C-band.

\section{Data used}

The study area is located in the west of the city of Algiers which is the capital of Algeria. It consists mainly of urban areas, agriculture fields and sea. The data was acquired on $11^{\text {th }}$ April 2009 by RADARSAT2 in a fully polarimetric mode in C-band. Table 1 gives more information about the acquisition and Fig. 1 shows the RGB image of the test site.

Table 1: Polarimetric data characteristics

\begin{tabular}{ll} 
Frequency & C-Band (5.3 GHz) \\
Polarization & HH, HV, VH, VV (Single Look \\
& Complex) \\
Resolution & $11 \times 9 \mathrm{~m}$ (azimuth) $*$ (distance) \\
Swath width & $25 \mathrm{~km}$ \\
Mode & Fine Quad-Pol \\
Date of acquisition & April $11^{\text {th } 2009}$ \\
Time of acquisition & $10: 54$ pm GMT \\
Orbit & Ascending, Ref : DT14 \\
Incidence angle & $38.34-39.81^{\circ}$ \\
\hline
\end{tabular}

\section{Full polarimetry theory}

The fundamental quantities measured by a polarimetric SAR are the scattering matrix elements $S_{t r}$, where $t$ and $r$ are the transmit and receive polarizations, respectively. The scattering matrix representation as defined in the linear $(\mathrm{H}$, V) basis is [8]:

$$
S=\left[\begin{array}{ll}
S_{h h} & S_{h v} \\
S_{h v} & S_{v v}
\end{array}\right]
$$

Rewriting this matrix in the equivalent vector in the Lexicographic basis yields:

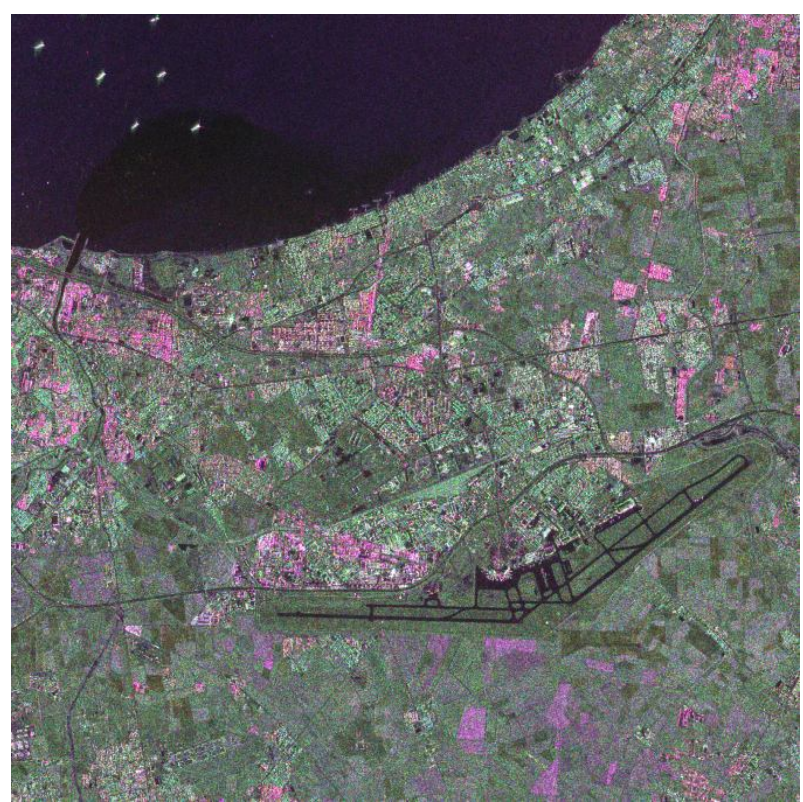

Figure 1: The RGB image of the polarimetric data of the test site (Red:HH, Green:HV and Blue:VV).

$$
\vec{k}=\left[\begin{array}{lll}
S_{h h} & \sqrt{2} S_{h v} & S_{v v}
\end{array}\right]^{T}
$$

Where the superscript $T$ indicates, the transpose operator.

The scattering vector in the Pauli basis is given as:

$$
\overrightarrow{k_{p}}=\frac{1}{\sqrt{2}}\left[\begin{array}{lll}
S_{h h}+S_{v v} & S_{h h}-S_{v v} & 2 S_{h v}
\end{array}\right]^{T}
$$

For multilook processed $3 \times 3$ positive semi-definite hermitian coherency and covariance matrices:

$$
\begin{aligned}
& \langle[T]\rangle=\left\langle\overrightarrow{k_{p} k_{p}} * T\right. \\
& \langle[C]\rangle=\left\langle\vec{k} \vec{k}^{* T}\right\rangle
\end{aligned}
$$

Where the superscript $* T$ denotes the conjugate transpose operator. The symbol $<>$ indicates ensemble averaging. The coherency matrix is related to the covariance matrix as [8]:

$$
\begin{gathered}
{[C]=\frac{1}{2}\left[\begin{array}{ccc}
1 & 1 & 0 \\
0 & 0 & \sqrt{2} \\
1 & -1 & 0
\end{array}\right][T]\left[\begin{array}{ccc}
1 & 0 & 1 \\
1 & 0 & -1 \\
0 & \sqrt{2} & 0
\end{array}\right]} \\
=\left[\begin{array}{ccc}
\left|S_{h h}\right|^{2} & \sqrt{2}\left(S_{h h} S_{h v}^{*}\right) & S_{h h} S_{v v}^{*} \\
\sqrt{2}\left(S_{h v} S_{h h}^{*}\right) & 2\left|S_{h v}\right|^{2} & \sqrt{2}\left(S_{h v} S_{v v}^{*}\right) \\
S_{v v} S_{h h}^{*} & \sqrt{2}\left(S_{v v} S_{h v}^{*}\right) & \left|S_{v v}\right|^{2}
\end{array}\right]
\end{gathered}
$$

In the case of a target characterized by reflection symmetry [9], the following relations hold:

$$
\left\langle S_{h h} S_{h v}^{*}\right\rangle=\left\langle S_{h v} S_{v v}^{*}\right\rangle=0
$$

Equations (5) can then be written as:

$$
[C]=\left[\begin{array}{ccc}
\left|S_{h h}\right|^{2} & 0 & S_{h h} S_{v v}^{*} \\
0 & 2\left|S_{h v^{2}}\right|^{2} & 0 \\
S_{v v} S_{h h}^{*} & 0 & \left|S_{v v}\right|^{2}
\end{array}\right]
$$




\section{Compact polarimetry theory}

Compact polarimetry is a technique that allows construction of pseudo quad-pol information from dual-polarization SAR data.

If a single polarization is transmitted, whereas the two canonical orthogonal linear polarizations $(\mathrm{H}$ and $\mathrm{V})$ are received, the 2-D measurement vector (or observable) $\vec{k}_{C P}$ is the projection of the full backscattering matrix $S$ on the transmit polarization state. The relation between $\vec{k}_{C P}$ and $S$ is given by [3]:

$$
\vec{k}_{C P}=S \vec{J}_{t}
$$

Where $\vec{J}_{t}$, represents the transmitted Jones vector. Table 2 provides examples of Jones vectors for canonical polarization states.

Table 2: Jones vector for canonical polarization states

\begin{tabular}{lllll}
\hline & \multicolumn{4}{c}{ Polarization cases } \\
& $\mathrm{H}$ & $\mathrm{V}$ & $\mathrm{RC}$ & $\mathrm{LC}$ \\
\hline$\vec{J}$ & $\left(\begin{array}{l}1 \\
0\end{array}\right)$ & $\left(\begin{array}{l}0 \\
1\end{array}\right)$ & $\frac{1}{\sqrt{2}}\left(\begin{array}{c}1 \\
-i\end{array}\right)$ & $\frac{1}{\sqrt{2}}\left(\begin{array}{l}1 \\
i\end{array}\right)$ \\
$(\psi, \chi)$ & $\left(0^{\circ}, 0^{\circ}\right)$ & $\left(90^{\circ}, 0^{\circ}\right)$ & $\left(\right.$ und, $\left.45^{\circ}\right)$ & $\left(\right.$ und,$\left.-45^{\circ}\right)$ \\
\hline
\end{tabular}

In this table $(\psi, \chi)$ stands for orientation and ellipticity angles, RC and LC for Right and Left circular respectively.

Und means undefined

The scattering vectors $\vec{k}_{C P}$ for the $\pi / 4$, dual circular polarimetric (DCP), and right circular transmit, linear (horizontal and vertical) receive or hybrid (CTLR) modes are given in Table 3 [5].

Table 3: Compact polarimetry modes

\begin{tabular}{|c|c|c|}
\hline Mode & Trans/Recep & $\vec{k}_{C P}$ \\
\hline$\pi / 4$ & $45^{\circ} /(\mathrm{H}, \mathrm{V})$ & {$\left[\begin{array}{ll}S_{h h}+S_{h v} & S_{v v}+S_{h v}\end{array}\right]^{T} / \sqrt{2}$} \\
\hline DCP & $\mathrm{RC} /(\mathrm{RC}, \mathrm{LC})$ & $\begin{array}{l}{\left[S_{R R} \quad S_{R L}\right]^{T}=} \\
{\left[S_{h h}-S_{v v}+i 2 S_{h v} \quad i\left(S_{h h}+S_{v v}\right)\right]^{T} /} \\
2\end{array}$ \\
\hline CTLR & $\mathrm{RC} /(\mathrm{H}, \mathrm{V})$ & {$\left[S_{h h}-i S_{h v}-i S_{v v}+S_{h v}\right]^{T} / \sqrt{2}$} \\
\hline
\end{tabular}

In this table, $45^{\circ}$ stands for linear polarization with a $45^{\circ}$ inclination.

Fig. 2 shows the different configurations of the CP modes.

The two vectors $\vec{k}_{D C P}$ and $\vec{k}_{C T L R}$ are related by the following equation [10].

$$
\vec{k}_{D C P}=\frac{1}{\sqrt{2}}\left[\begin{array}{cc}
1 & -i \\
1 & i
\end{array}\right] \vec{k}_{C T L R}
$$

The two vectors have equivalent polarimetric information. This case is important because it means that the analysis of the data from these two modes should generally lead to the same results.

The measured compact polarimetric vector $\vec{k}_{c p}$ and its corresponding covariance matrix $C_{c p}$ are given by:

$$
\vec{k}_{c p}=\left[\begin{array}{ll}
C P_{1} & C P_{2}
\end{array}\right]^{T}
$$

$$
\left[C_{c p}\right]=\left\langle\vec{k}_{c p} \vec{k}_{c p}^{* T}\right\rangle=\left[\begin{array}{ll}
C_{11} & C_{12} \\
C_{21} & C_{22}
\end{array}\right]
$$

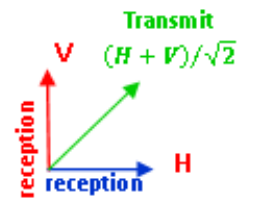

(a)
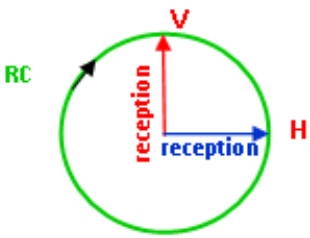

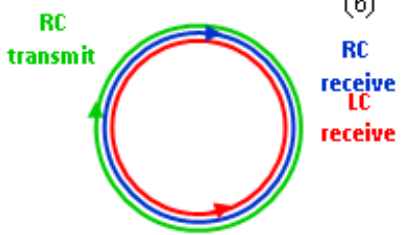

(c)

Figure 2: The three configurations of the compact polarimetry (a) $\pi / 4$, (b) CTLR and (c) DCP.

Where $C_{11}=\left\langle\left|C P_{1}\right|^{2}\right\rangle, C_{12}=C_{12}{ }^{*}=\left\langle C P_{1} * C P_{2}{ }^{*}\right\rangle$ and $C_{22}=\left\langle\left|C P_{2}\right|^{2}\right\rangle \cdot C_{c p}$, is Hermitian and provides four measurements, two real diagonal terms and the real and imaginary parts of one of the off-diagonal terms.

The relevant $2 \times 2$ Hermitian covariance matrices become:

$\left[C_{\frac{\pi}{4}}\right]=\left\langle\vec{k}_{\frac{\pi}{4}} \vec{k}_{\frac{\pi}{4}}^{* T}\right\rangle=$

$\frac{1}{2}\left[\begin{array}{cc}\left\langle\left|S_{h h}\right|^{2}\right\rangle & \left\langle S_{h h} S_{v v}^{*}\right\rangle \\ \left\langle S_{v v} S_{h h}^{*}\right\rangle & \left\langle\left|S_{v v}\right|^{2}\right\rangle\end{array}\right]+\frac{1}{2}\left\langle\left|S_{h v}\right|^{2}\right\rangle\left[\begin{array}{ll}1 & 1 \\ 1 & 1\end{array}\right]+$

$\frac{1}{2}\left[\begin{array}{cc}2 \Re\left(\left\langle S_{h h} S_{h v}^{*}\right\rangle\right) & \left\langle S_{h h} S_{h v}^{*}\right\rangle+\left\langle S_{h v} S_{v v}^{*}\right\rangle \\ \left\langle S_{h h}^{*} S_{h v}\right\rangle+\left\langle S_{v v} S_{h v}^{*}\right\rangle & 2 \mathfrak{R}\left(\left\langle S_{v v} S_{h v}^{*}\right\rangle\right)\end{array}\right]$

$\left[C_{D C P}\right]=\left\langle\vec{k}_{D C P} \vec{k}_{D C P}{ }^{* T}\right\rangle$

$=\frac{1}{4}\left[\begin{array}{cc}\left\langle\left|S_{h h}-S_{v v}\right|^{2}\right\rangle & \left\langle-i\left(S_{h h}-S_{v v}\right)\left(S_{h h}+S_{v v}\right)^{*}\right\rangle \\ \left\langle-i\left(S_{h h}+S_{v v}\right)\left(S_{h h}-S_{v v}\right)^{*}\right\rangle & \left\langle\left|S_{h h}+S_{v v}\right|^{2}\right\rangle\end{array}\right]$

$+\frac{1}{4}\left[\begin{array}{cc}4\left\langle\left|S_{h v}\right|^{2}\right\rangle & 0 \\ 0 & 0\end{array}\right]+$

$\frac{1}{4}\left[\begin{array}{cc}4\left\langle\Im\left(\left(S_{h h}-S_{v v}\right) \cdot S_{h v}^{*}\right)\right\rangle & 2\left\langle\left(S_{h h}+S_{v v}\right)^{*}\right\rangle \\ 2\left\langle\left(S_{h h}+S_{v v}\right) \cdot S_{h v}^{*}\right\rangle & 0\end{array}\right]$

$\left[C_{C T L R}\right]=\left\langle\vec{k}_{C T L R} \vec{k}_{C T L R}{ }^{* T}\right\rangle$

$=\frac{1}{2}\left[\begin{array}{cc}\left\langle\left|S_{h h}\right|^{2}\right\rangle & \left\langle i\left(S_{h h} S_{v v}^{*}\right)\right\rangle \\ \left\langle-i\left(S_{v v} S_{h h}^{*}\right)\right\rangle & \left\langle\left|S_{v v}\right|^{2}\right\rangle\end{array}\right]+\frac{1}{2}\left\langle\left|S_{h v}\right|^{2}\right\rangle\left[\begin{array}{cc}1 & -i \\ i & 1\end{array}\right]$

$+\frac{1}{2}\left[\begin{array}{cc}-2 \mathfrak{I}\left(\left\langle S_{h h} S_{h v}^{*}\right\rangle\right) & \left\langle S_{h h} S_{h v}^{*}\right\rangle+\left\langle S_{h v} S_{v v}^{*}\right\rangle \\ \left\langle S_{h h}^{*} S_{h v}\right\rangle+\left\langle S_{v v} S_{h v}^{*}\right\rangle & 2 \mathfrak{J}\left(\left\langle S_{v v} S_{h v}^{*}\right\rangle\right)\end{array}\right]$

The resulting compact polarimetry covariance matrices are expressed as a sum of three terms. The first term contains elements that depend only on $S_{h h}$ and $S_{v v}$, the second term contains $\left\langle\left|S_{h v}\right|^{2}\right\rangle$ elements, and the last term consists only of co-polarization (co-pol) and cross-pol correlations. 
We come up with an undetermined system of four equations (linked to the two real measurements $C_{11}, C_{22}$ and the complex one $C_{12}$ ) and six variables:

$$
\begin{array}{ll}
\mathrm{H}=\left\langle\left|S_{h h}\right|^{2}\right\rangle, & V=\left\langle\left|S_{v v}\right|^{2}\right\rangle, \quad \mathrm{X}=\left\langle\left|S_{h v}\right|^{2}\right\rangle, \\
\mathrm{P}=\left\langle S_{h h} S_{v v}^{*}\right\rangle, & \left.\left\langle S_{h h} S_{h v}^{*}\right\rangle \quad \text { and }\left\langle S_{v v} S_{h v}^{*}\right\rangle\right) .
\end{array}
$$

Additional information is, therefore required to solve it. For this reason, two hypotheses related to the polarimetric behavior of the compact covariance matrix components have been introduced [6].

- The first one suppose reflection symmetry as stated in equation (6).

- The second assumption relates the co-pol correlation coefficient to the relative magnitudes of the cross-pol and co-pol responses.

$$
\frac{X}{H+V} \approx \frac{1-\left|\rho_{h-v}\right|}{4}
$$

Where $\rho_{h-v}=\frac{\left\langle S_{h h} S_{v v}^{*}\right\rangle}{\sqrt{\left.\left.\left\langle S_{h h^{2}}\right\rangle \cdot|| S_{v v}\right|^{2}\right\rangle}}$ is the linear correlation between $S_{h h}$ and $S_{v v}$.

The assumption of reflection symmetry implies that the last term is null and the covariance matrices become:

$$
\begin{aligned}
{\left[C_{\frac{\pi}{4}}\right]=\left\langle\vec{k}_{\frac{\pi}{4}} \vec{k}_{\frac{\pi}{4}}^{* T}\right\rangle } & =\frac{1}{2}\left[\begin{array}{cc}
\left\langle\left. S_{h h}\right|^{2}\right\rangle & \left\langle S_{h h} S_{v v}^{*}\right\rangle \\
\left\langle S_{v v} S_{h h}^{*}\right\rangle & \left\langle\left|S_{v v}\right|^{2}\right\rangle
\end{array}\right] \\
& +\frac{1}{2}\left\langle\left|S_{h v}\right|^{2}\right\rangle\left[\begin{array}{ll}
1 & 1 \\
1 & 1
\end{array}\right]
\end{aligned}
$$

$\left[C_{D C P}\right]=\left\langle\vec{k}_{D C P} \vec{k}_{D C P}{ }^{* T}\right\rangle$

$$
\begin{gathered}
\frac{1}{4}\left[\begin{array}{cc}
\left\langle\left|S_{h h}-S_{v v}\right|^{2}\right\rangle & \left\langle-i\left(S_{h h}-S_{v v}\right)\left(S_{h h}+S_{v v}\right)^{*}\right\rangle \\
\left\langle-i\left(S_{h h}+S_{v v}\right)\left(S_{h h}-S_{v v}\right)^{*}\right\rangle & \left\langle\left|S_{h h}+S_{v v}\right|^{2}\right\rangle
\end{array}\right] \\
+\frac{1}{4}\left[\begin{array}{cc}
4\left\langle\left|S_{h v}\right|^{2}\right\rangle & 0 \\
0 & 0
\end{array}\right]
\end{gathered}
$$

$\left[C_{C T L R}\right]=\left\langle\vec{k}_{C T L R} \vec{k}_{C T L R}{ }^{* T}\right\rangle$

$=\frac{1}{2}\left[\begin{array}{cc}\left\langle\mid S_{h h^{2}}\right\rangle & \left\langle i\left(S_{h h} S_{v v}^{*}\right)\right\rangle \\ \left\langle-i\left(S_{v v} S_{h h}^{*}\right)\right\rangle & \left\langle\left|S_{v v}\right|^{2}\right\rangle\end{array}\right]+\frac{1}{2}\left\langle\left|S_{h v}\right|^{2}\right\rangle\left[\begin{array}{cc}1 & -i \\ i & 1\end{array}\right]$

Here, each mode reduces to a system of four equations from the covariance matrix and five unknowns. For the $\pi / 4$ and CTLR modes, the unknowns are $\left|S_{h h}\right|^{2},\left|S_{v v}\right|^{2},\left|S_{h v}\right|^{2}$, $\left(S_{h h} S_{v v}^{*}\right)$ and $\left(S_{v v} S_{h h}^{*}\right)$, where the last unknown is complex. As stated in equation (17), the DCP mode depends on the Pauli basis coefficients $\left(S_{h h}-S_{v v}\right),\left(S_{h h}+S_{v v}\right)$, and $S_{h v}$.

The assumption of reflection symmetry implies that the last term is null and the covariance matrices become:

$$
\begin{gathered}
{\left[C_{\frac{\pi}{4}}\right]=\left\langle\vec{k} \frac{\pi}{4} \vec{k}_{\frac{\pi}{4}}^{* T}\right\rangle=\frac{1}{2}\left[\begin{array}{cc}
\left\langle\left|S_{h h}\right|^{2}\right\rangle & \left\langle S_{h h} S_{v v}^{*}\right\rangle \\
\left\langle S_{v v} S_{h h}^{*}\right\rangle & \left\langle\left|S_{v v}\right|^{2}\right\rangle
\end{array}\right]+} \\
\frac{1}{2}\left\langle\left|S_{h v}\right|^{2}\right\rangle\left[\begin{array}{cc}
1 & 1 \\
1 & 1
\end{array}\right] \\
\frac{1}{4}\left[\begin{array}{cc}
{\left[C_{D C P}\right]=\left\langle\vec{k}_{D C P} \vec{k}_{D C P}{ }^{* T}\right\rangle=} \\
\left\langle\left|S_{h h}-S_{v v}\right|^{2}\right\rangle & \left\langle-i\left(S_{h h}-S_{v v}\right)\left(S_{h h}+S_{v v}\right)^{*}\right\rangle \\
\left\langle-i\left(S_{h h}+S_{v v}\right)\left(S_{h h}-S_{v v}\right)^{*}\right\rangle & \left\langle\left|S_{h h}+S_{v v}\right|^{2}\right\rangle
\end{array}\right] \\
+\frac{1}{4}\left[\begin{array}{cc}
4\left\langle\left|S_{h v}\right|^{2}\right\rangle & 0 \\
0 & 0
\end{array}\right]
\end{gathered}
$$

$$
\begin{gathered}
{\left[C_{C T L R}\right]=\left\langle\vec{k}_{C T L R} \vec{k}_{C T L R}{ }^{* T}\right\rangle} \\
=\frac{1}{2}\left[\begin{array}{cc}
\left\langle\left|S_{h h}\right|^{2}\right\rangle & \left\langle i\left(S_{h h} S_{v v}^{*}\right)\right\rangle \\
\left\langle-i\left(S_{v v} S_{h h}^{*}\right)\right\rangle & \left\langle\left|S_{v v}\right|^{2}\right\rangle
\end{array}\right]+\frac{1}{2}\left\langle\left|S_{h v}\right|^{2}\right\rangle\left[\begin{array}{cc}
1 & -i \\
i & 1
\end{array}\right]
\end{gathered}
$$

\section{Pseudo quad-pol reconstruction algorithm}

The construction of the pseudo quad-pol covariance matrices from the compact polarimetry modes is based on a pair of equations that are iteratively solved for $\left\langle\left|S_{h v}\right|^{2}\right\rangle$ [6].

\section{1. $\pi / 4$ mode solution}

For the $\pi / 4$ mode, the solution of equations starts with the initial values of $\left\langle\left|S_{h v}\right|^{2}\right\rangle$ and the linear co-polarization coherence $\rho=\rho_{h-v}$.

$$
\begin{aligned}
& \rho_{(0)}=\frac{C_{12}}{\sqrt{C_{11} C_{22}}} \\
& \left\langle\left|S_{h v}\right|^{2}\right\rangle_{(0)}=\frac{C_{11}+C_{22}}{2}\left(\frac{1-\left|\rho_{(0)}\right|}{3-\left|\rho_{(0)}\right|}\right)
\end{aligned}
$$

and then iterates the following equations:

$$
\begin{aligned}
& \rho_{(i+1)}=\frac{C_{12}-\left\langle\left|S_{h v}\right|^{2}\right\rangle_{(i)}}{\sqrt{\left(C_{11}-\left\langle\mid S_{h v^{2}}\right\rangle_{(i)}\right)\left(C_{22}-\left\langle\left|S_{h v^{2}}\right|_{(i)}\right)\right.}} \\
& \left\langle\left|S_{h v}\right|^{2}\right\rangle_{(i+1)}=\frac{C_{11}+C_{22}}{2}\left(\frac{1-\left|\rho_{(i+1)}\right|}{3-\left|\rho_{(i+1)}\right|}\right)
\end{aligned}
$$

Given a value for $\left\langle\left|S_{h v}\right|^{2}\right\rangle=\left\langle\left|S_{h v}\right|^{2}\right\rangle_{(n)}$ (where $\mathrm{n}$ is the order of iteration), the pseudo quad-pol covariance matrix is then constructed by:

$$
\begin{aligned}
& {[C]_{\text {pseudo quad }}=} \\
& {\left[\begin{array}{ccc}
C_{11}-\left\langle\left|S_{h v}\right|^{2}\right\rangle & 0 & C_{12}-\left\langle\left|S_{h v}\right|^{2}\right\rangle \\
0 & 2\left\langle\left|S_{h v}\right|^{2}\right\rangle & 0 \\
\left(C_{12}-\left\langle\left|S_{h v}\right|^{2}\right\rangle\right)^{*} & 0 & C_{22}-\left\langle\left|S_{h v}\right|^{2}\right\rangle
\end{array}\right]}
\end{aligned}
$$

The null components are the characteristic of the reflection symmetry assumption.

\subsection{DCP mode solution}

The iterative equations and pseudo quad-pol covariance matrix for the DCP mode are [5]:

$$
\begin{aligned}
& \rho_{(i+1)}=\frac{C_{12}+\left\langle\mid S_{h v^{2}}\right\rangle_{(i)}}{\sqrt{\left(C_{11}-\left\langle\mid S_{h v^{\prime}}\right\rangle_{(i)}\right)\left(C_{22}-\langle| S_{\left.\left.h v^{\prime 2}\right\rangle_{(i)}\right)}\right.}} \\
& \left\langle\left|S_{h v}\right|^{2}\right\rangle_{(i+1)}=\frac{C_{11}+C_{22}}{2}\left(\frac{1-\left|\rho_{(i+1)}\right|}{3-\left|\rho_{(i+1)}\right|}\right)
\end{aligned}
$$

$[C]_{p s e u d o ~ q u a d}=$

$$
\left[\begin{array}{ccc}
C_{11}-\left\langle\left|S_{h v}\right|^{2}\right\rangle & 0 & C_{12}+\left\langle\left|S_{h v}\right|^{2}\right\rangle \\
0 & 2\left\langle\left|S_{h v}\right|^{2}\right\rangle & 0 \\
\left(C_{12}+\left\langle\left|S_{h v}\right|^{2}\right\rangle\right)^{*} & 0 & C_{22}-\left\langle\left|S_{h v}\right|^{2}\right\rangle
\end{array}\right]
$$


$\left\langle\left|S_{h v}\right|^{2}\right\rangle=\left\langle\left|S_{h v}\right|^{2}\right\rangle_{(n)}$

\subsection{CTLR mode solution}

The construction of the pseudo quad-pol covariance matrices CTLR mode is similar to the previous mode. The iterative equations and pseudo quad-pol covariance matrix for the CTLR mode are [5]:

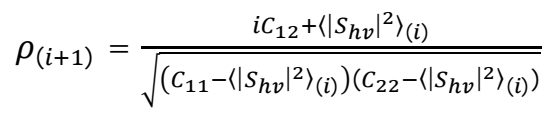

$$
\begin{aligned}
& \left\langle\left|S_{h v}\right|^{2}\right\rangle_{(i+1)}=\frac{C_{11}+C_{22}}{2}\left(\frac{1-\left|\rho_{(i+1)}\right|}{3-\left|\rho_{(i+1)}\right|}\right) \\
& {[C]_{\text {pseudo quad }}} \\
& =\left[\begin{array}{ccc}
C_{11}-\left\langle\left|S_{h v}\right|^{2}\right\rangle & 0 & i C_{12}+\left\langle\left|S_{h v}\right|^{2}\right\rangle \\
0 & 2\left\langle\left|S_{h v}\right|^{2}\right\rangle & 0 \\
\left(i C_{12}+\left\langle\left|S_{h v}\right|^{2}\right\rangle\right)^{*} & 0 & C_{22}-\left\langle\left|S_{h v}\right|^{2}\right\rangle
\end{array}\right] \\
& \left\langle\left|S_{h v}\right|^{2}\right\rangle=\left\langle\left|S_{h v}\right|^{2}\right\rangle_{(n)} .
\end{aligned}
$$

For all the three modes, it occurs that $\rho_{(n)}$ may become larger than one for certain pixels, or even that its denominator becomes the square root of a negative number. In both cases, we regularize by setting $\rho_{(n)}=1$ and $\left\langle\mid S_{h v}{ }^{2}\right\rangle_{(n)}=0$ and then halt the iteration.

Using CTLR mode would involve the same properties as those established hereafter with transmitted linear polarization [6]. The two vectors of CTLR and DCP modes have also equivalent polarimetric information, so they should generally lead to the same results. Therefore, we will limit our discussion in this paper to the $\pi / 4$ mode.

\subsection{Modification of the compact polarimetry reconstruction algorithm}

The second assumption proposed by souyris et $a l$. may not always hold [5]. Hence, an alternate method has been set out for approximating the value of $\mathrm{X}$ in order to derive better pseudo quad-pol representations. The construction algorithms employ for iteratively updating X:

$$
\left\langle\left|S_{h v}\right|^{2}\right\rangle_{(i+1)}=\left(C_{11}+C_{22}\right)\left(\frac{1-\left|\rho_{(i+1)}\right|}{N+2\left(1-\left|\rho_{(i+1)}\right|\right)}\right)
$$

Where $N=\left(\left|S_{h h}-S_{v v}\right|^{2}\right) /\left|S_{h v}\right|^{2}$

The value of $\mathrm{N}$ is estimated from the resulting original reconstruction algorithm.

To show the performance of the reconstructed algorithm, a full quad-pol data set was used to generate the CP mode, and then the corresponding pseudo quad-pol data sets were constructed via (21)-(33) equations. The datasets used to illustrate the $\mathrm{CP}$ mode are the RADARSAT2 C-band fully polarimetric images of the west region of the Algiers city and an agriculture field region situated in the south of the same town. It contains symmetric and non-symmetric scattering SAR targets.

\section{Linear co-polarization coherence $\left|\rho_{h-v}\right|$ mapping}

\subsection{Linear co-polarization coherence $\left|\rho_{h-v}\right|$ mapping}

Fig. 3 displays the mapping of the degree of coherence $\left|\rho=\rho_{h-v}\right|$ for consecutive orders of estimation. The test is conducted on the same zone which is the west region of Algiers, with a $5 \times 5$ analysis window. Fig. 3.a displays the FP reference value. The first order estimate shown in Fig. 3.b produces a "milky" impression, which alters the image contrast. However, the third estimated coherence shown in Fig. 3.c is qualitatively very close to the FP coherence.

Fig. 3d displays the last estimate of the reconstructed degree coherence versus the actual degree of coherence. As expected, this figure shows very good reconstruction performances.

\subsection{Quantitative assessment}

For comparison, the original FP data are shown in Fig. 4a with $\mathrm{H}$ in red, $\mathrm{X}$ in green, and $\mathrm{V}$ in blue. The $\pi / 4$ mode synthesized result is shown in Fig. $4 \mathrm{~b}$. We notice some differences between these two images, especially in the $\mathrm{X}$ intensity in some urban areas, which is noticeably lower for the $\mathrm{CP}$ mode. However, strong similarity does exist in polarimetric response of most of the rest of the test area.

Fig. 5 shows scatter plots detailing how well the derived pseudo-quad-pol results fit the original quad-pol. It shows also the performance of the reconstruction algorithm. Most of the points of the scatter plot fall close the one-to-one line with small spread. A small systematic overestimation of the $\left|S_{h v}\right|^{2}$ can be observed over this data. For every channel, an overall agreement is observed between the reconstructed and actual radiometric values.

\subsection{Compact polarimetric signatures}

The concept of the polarization signature of a scatterer was used by Van zyl et al. [11] to graph the power of a return wave as a function of transmit and receive polarizations. The backscattering radar cross section is given by:

$$
\sigma(\psi, \chi)=\frac{4 \pi}{k^{2}} \overrightarrow{S_{r}}[K] \overrightarrow{S_{t}}
$$

Recall that $t$ and $r$ denote the received and the transmitted polarizations. $k$ is the transmitted wavenumber, $\psi$ and $\chi$ are the orientation and the ellipticity angles. The first angle $(\psi)$ ranges between $0^{\circ}$ to $180^{\circ}$ and the second one $(\chi)$ is defined between $-45^{\circ}$ to $45^{\circ}$.

$\overrightarrow{S_{l}}$ is the normalized Stokes vector defined as:

$$
\overrightarrow{S_{l}}=\left[\begin{array}{c}
1 \\
\cos \left(2 \psi_{i}\right) \sin \left(2 \chi_{i}\right) \\
\sin \left(2 \psi_{i}\right) \cos \left(2 \chi_{i}\right) \\
\sin \left(2 \chi_{i}\right)
\end{array}\right]
$$

The subscript $i$ denotes $r$ or $t$.

The elements of the Kennaugh matrix $[K]$ can be calculated 


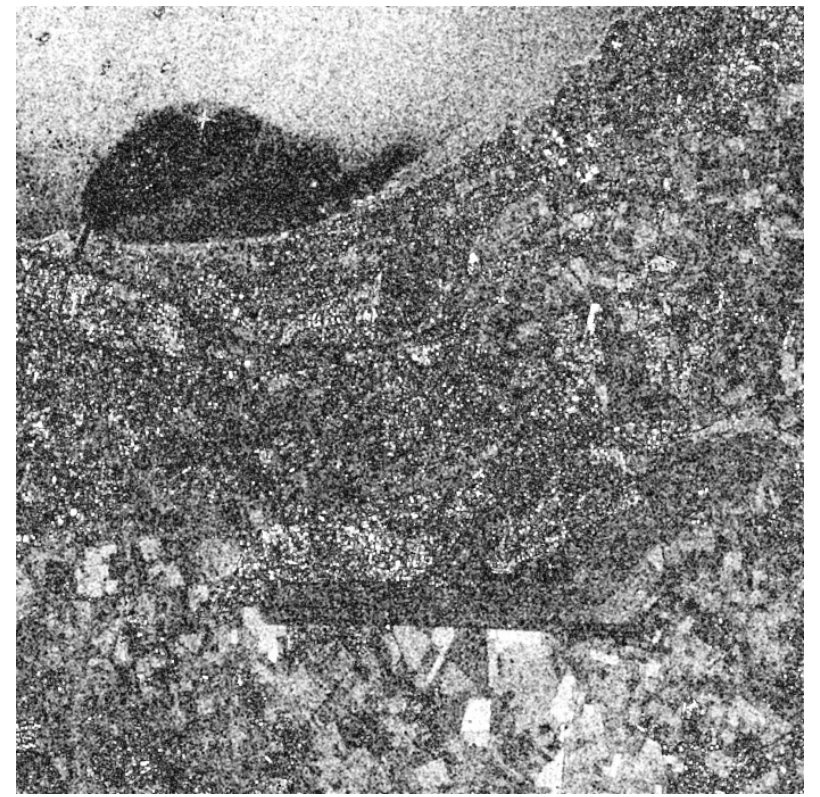

(a)

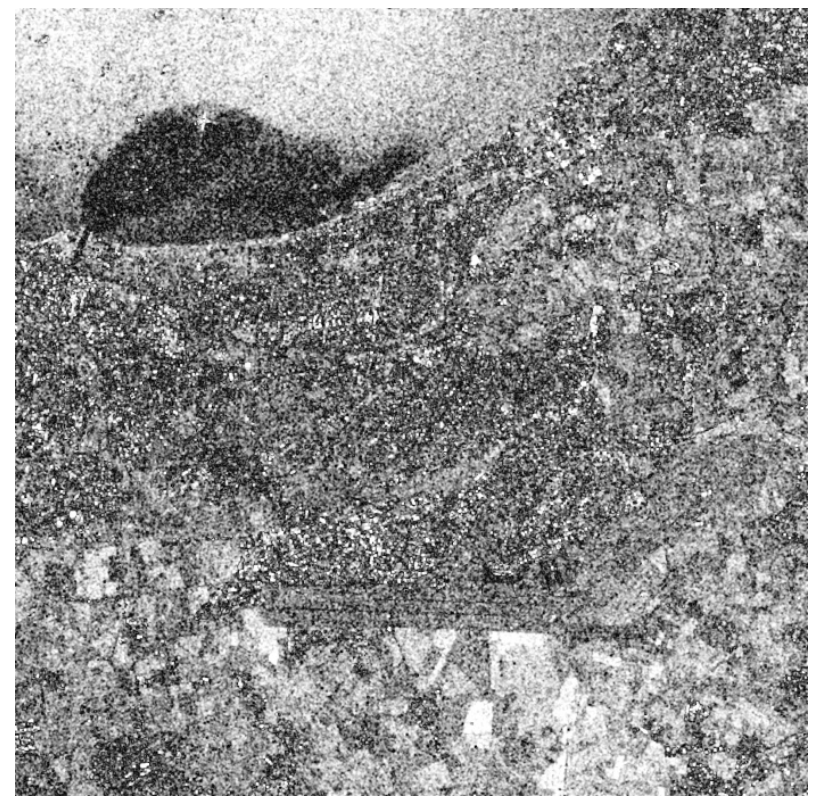

(c)

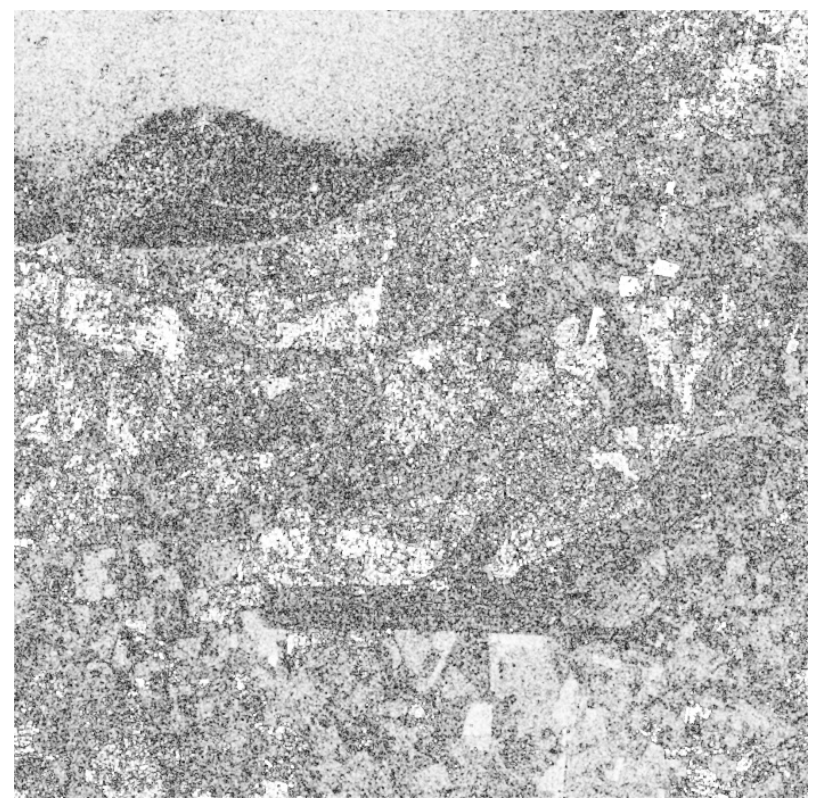

(b)

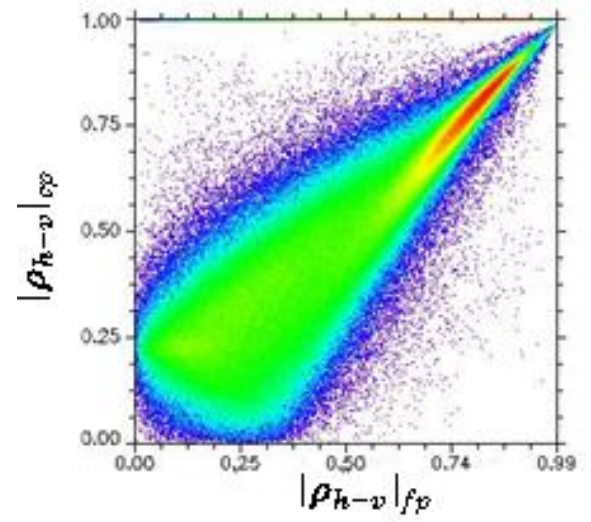

(d)

Figure 3: Degree of coherence $\left|\rho=\rho_{h-v}\right|$, (a) reference value of $\left|\rho_{h-v}\right|$ inferred from FP. ( $0=$ black, $1=$ white), (b)-(c) first- and second-order estimates of $\left|\rho_{h-v}\right|$, (d) reconstructed degree of coherence ( $3^{\text {rd }}$ order estimates) versus actual degree of coherence (reference value )

from the coherency matrix $[T]$ as follows [2]

$$
k_{i j}=\frac{1}{2} \operatorname{tr}\left([T] \eta_{i j}\right)
$$

With $\eta_{i j}=(-1)^{-\delta_{i j}[A] \sigma_{i} \otimes \sigma_{j}[A]^{* T}}$

Where $[A]=\frac{1}{\sqrt{2}}\left[\begin{array}{cccc}1 & 0 & 0 & 1 \\ 1 & 0 & 0 & -1 \\ 0 & 1 & 1 & 0 \\ 0 & i & -i & 0\end{array}\right]$ and $\delta_{i j}=\left\{\begin{array}{cc}0 & i \neq j \\ 1 & i=j\end{array}\right.$

$\sigma_{i, j}=(i, j=0,1,2,3)$ are the four Pauli matrices given by $\sigma_{0}=\left[\begin{array}{ll}1 & 0 \\ 0 & 1\end{array}\right], \quad \sigma_{1}=\left[\begin{array}{cc}1 & 0 \\ 0 & -1\end{array}\right], \quad \sigma_{2}=\left[\begin{array}{ll}0 & 1 \\ 1 & 0\end{array}\right]$, and $\sigma_{3}=$
$\left[\begin{array}{cc}0 & -i \\ i & 0\end{array}\right]$

The symbol $\otimes$ denotes the standard tensorial Kronecker matrix product.

One collects coherent CP SAR imagery by transmitting a given polarized signal and then coherently receiving any pair of orthogonal polarizations. While the transmitted polarization is fixed, from the coherently received signals one may synthesize the response of any receive polarization. 


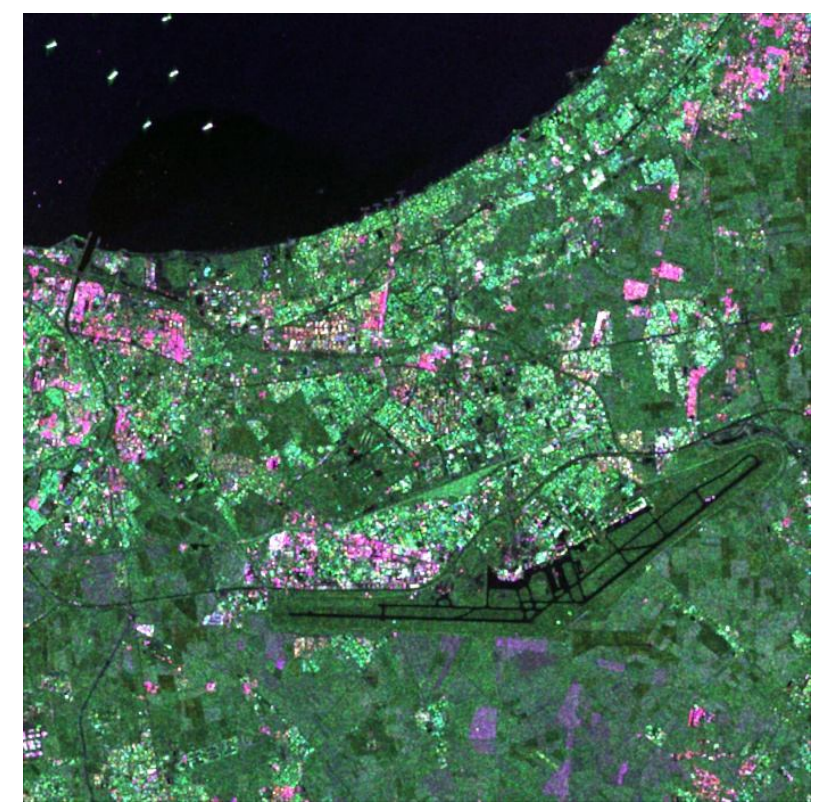

(a)

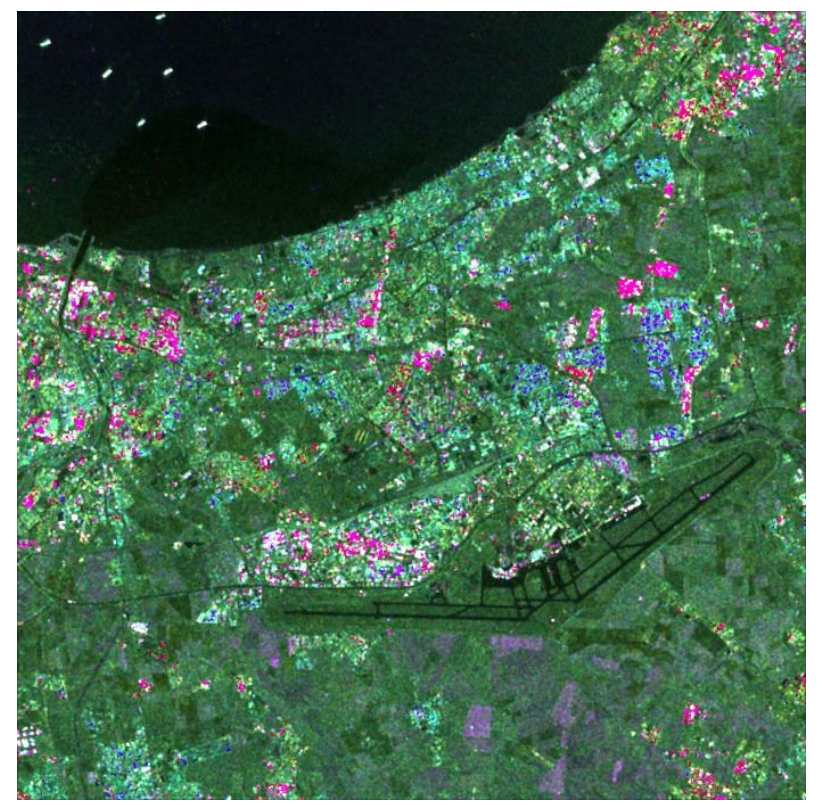

(b)

Figure 4: Full (a) RGB versus compact polarimetric (b) RGB color composit images, (Red:H, Green:X, Blue:V)

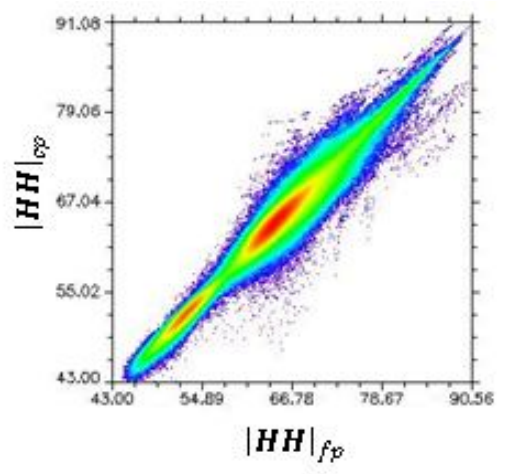

(a)

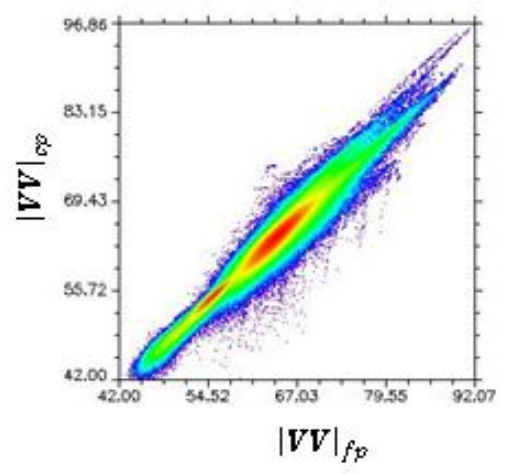

(b)

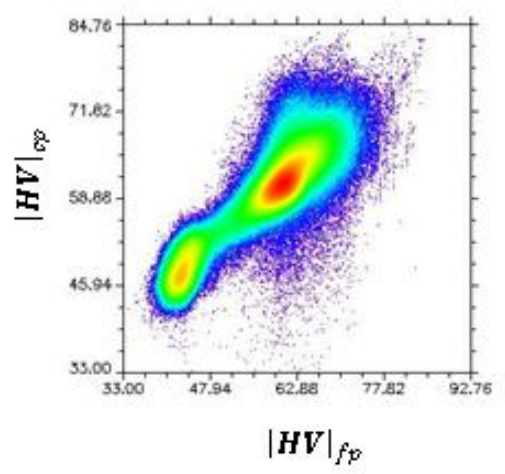

(c)

Figure 5 : Reconstruction performance for $\mathrm{H}, \mathrm{V}$ and $\mathrm{X}$ channels. Scatter plots of (a) $H_{\mathrm{fp}}-H_{\mathrm{CP}}$, (b) $V_{\mathrm{fp}}-V_{\mathrm{cp}}$ and (c) $X_{\mathrm{fp}}-X_{\mathrm{cp}}$

Therefore, the received power, as a function of the ellipticity and orientation angles of the received polarization, completely characterizes the $\mathrm{CP}$ response.

The two-dimensional surface plot of the received power as a function of polarization ellipticity and orientation provides a simple, graphical way to display this result.

Plots of the $\mathrm{CP}$ response from a variety of scattering mechanisms, e.g. rough surface, dihedral, dipole, allow for an easy visual analysis of the $\mathrm{CP}$ information. The results for the selected subregions shown in Fig. 6 that consist primarily of sea, grass field and urban structure are given in Fig. 7 and Fig. 8 where the quad- and CP.
Signatures of surface scattering and dihedral scattering representing three subregions were plotted and compared against each other.

Of note in these figures is that employing the CP- and quadpol signatures yield essentially the same results.

As shown in these figures, the urban targets have the double-bounce reflection properties and the sea and the grass fields have the surface scattering characteristics.

\subsection{Three component decomposition}

Freeman and Durden [12] introduced an unsupervised classification based on a three-component scattering model under the well-known reflection symmetry condition using 


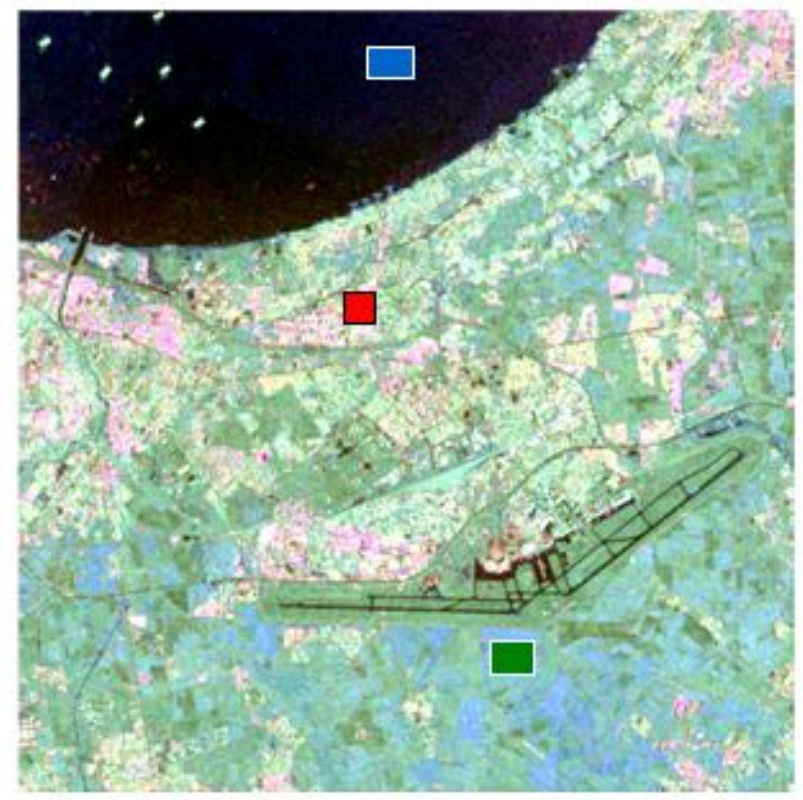

Figure 6: The selected regions of interests to generate the polarimetric signatures in full and compact modes. The three outlined areas the urban (double-bounce scattering), grass (surface scattering), and sea (Bragg surface) regions over which the polarimetric signatures are examined.

the covariance matrix. The components of the scattering matrix are analyzed to assign each pixel to one of three scattering categories of the model: surface, double-bounce scattering, and volume scattering. The mechanisms are a

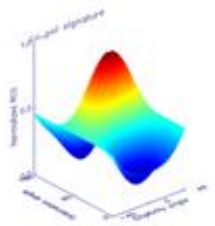

(a)
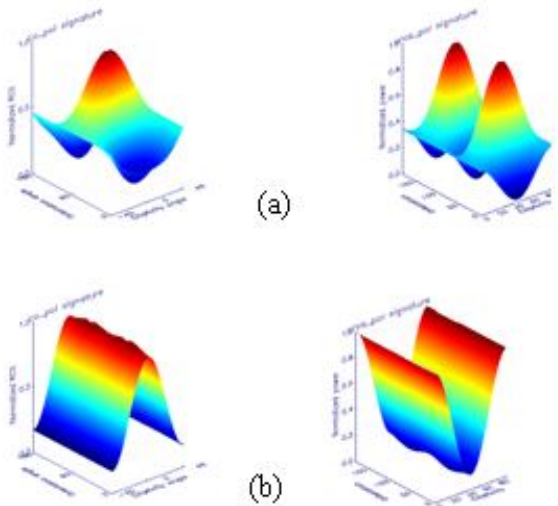

(b)
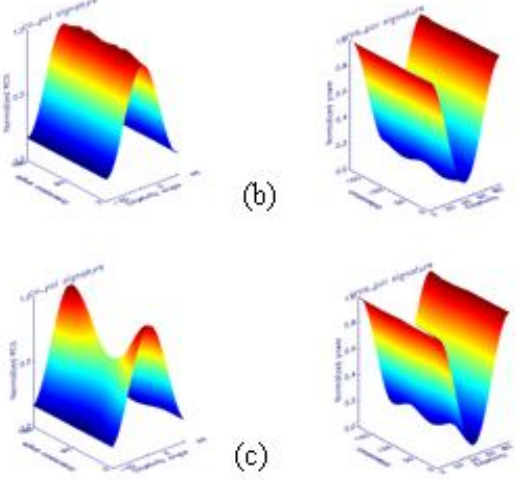

(c)

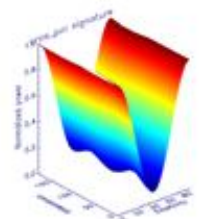

Figure 7: Co- and cross-polarimetric signatures using full polarimetric mode (a) urban region, (b) Agriculture field region, and (c) sea region. canopy scatter from a cloud of randomly oriented dipoles, even- or double-bounce scatter from a pair of orthogonal surfaces with different dielectric constants, and Bragg scatter from a moderately rough surface.

The Freeman-Durden model for the scattering and covariance matrices are:

- For the scattering processes for slightly rough surfaces

$$
[S]=\left[\begin{array}{ll}
\beta & 0 \\
0 & 1
\end{array}\right] \Rightarrow\langle[C]\rangle_{S}=\left[\begin{array}{ccc}
|\beta|^{2} & 0 & \beta \\
0 & 0 & 0 \\
\beta^{*} & 0 & 1
\end{array}\right]
$$

The obtained covariance matrix terms are : $\left\langle\left|S_{h h}\right|^{2}\right\rangle=|\beta|^{2}$, $\left\langle\left|S_{v v}\right|^{2}\right\rangle=1,\left\langle S_{h h} S_{v v}^{*}\right\rangle=\beta,\left\langle\left|S_{h v}\right|^{2}\right\rangle=0$ and $\left\langle S_{h h} S_{h v}^{*}\right\rangle=$ $\left\langle S_{h v} S_{v v}^{*}\right\rangle=0$.

Where $\beta$ is a parameter related to the relative dielectric constant of the surface and the local orientation angle.

- For the double-bounce scattering

The scattering matrix and the corresponding covariance matrix are:

$$
[S]=\left[\begin{array}{ll}
\alpha & 0 \\
0 & 1
\end{array}\right] \Rightarrow\langle[C]\rangle_{d}=\left[\begin{array}{ccc}
|\alpha|^{2} & 0 & \alpha \\
0 & 0 & 0 \\
\alpha^{*} & 0 & 1
\end{array}\right]
$$

Where $\alpha$ is related to tree trunk and ground reflection coefficients for horizontal and vertical polarizations.

- For a cloud of identical particles with random orientations and very thin horizontal cylinders like needle scatterers, the volume scattering averaged covariance matrix is given by

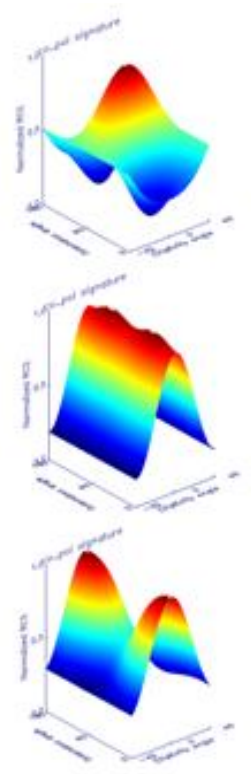

(a)

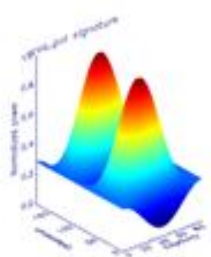

(b)

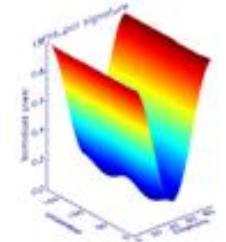

(c)

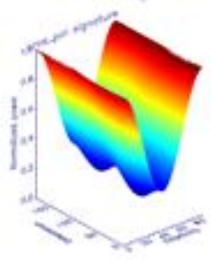

Figure 8: Co- and cross-polarimetric signatures using compact polarimetric mode (a) urban region, (b) Agriculture field region, and (c) sea region. 


$$
\langle[C]\rangle_{v}=\left[\begin{array}{ccc}
1 & 0 & 1 / 3 \\
0 & 2 / 3 & 0 \\
1 / 3 & 0 & 1
\end{array}\right]
$$

Finally, the covariance matrix of these models of the decomposition has the following form:

$$
\langle[C]\rangle=f_{s}\langle[C]\rangle_{s}+f_{d}\langle[C]\rangle_{d}+f_{v}\langle[C]\rangle_{v}
$$

Where $f_{s}, f_{d}$, and $f_{v}$, are the coefficients of the surface, double-bounce and volume scattering respectively.

The model for the total backscatter is

$$
\langle[C]\rangle=\left[\begin{array}{ccc}
C_{11} & 0 & C_{13} \\
0 & 0 & 0 \\
C_{13}^{*} & 0 & C_{33}
\end{array}\right]
$$

Where $C_{11}=f_{s}|\beta|^{2}+f_{d}|\alpha|^{2}+\frac{3}{8} f_{v}$,

$C_{12}=f_{s} \beta+f_{d} \alpha+\frac{1}{8} f_{v}, C_{33}=f_{s}+f_{d}+\frac{3}{8} f_{v}$

Finally, we estimate the contribution of each scattering mechanism to the span

$$
\begin{aligned}
\text { span } & =\left|S_{h h^{2}}\right|^{2}+2\left|S_{h v}\right|^{2}+\left|S_{v v}\right|^{2} \\
& =P_{S}+P_{d}+P_{v}
\end{aligned}
$$

With $P_{s}=f_{s}\left(1+|\beta|^{2}\right), P_{d}=f_{d}\left(1+|\alpha|^{2}\right)$ and $P_{v}=f_{v}$

Fig. 9a and 9b shows the result of applying the FreemanDurden decomposition to the polarimetric data. In each figure, the contributions of each of the three scattering mechanisms to the total power are shown for each pixel, with surface scatter colored blue, volume scatter green, and double-bounce red. The relative strength of each color in the resulting RGB images can be related to the relative strengths of the scattering mechanisms.

The blue areas are dominated by single-bounce surface, or grass, backscatter whereas the red areas are dominated by double-bounce backscatter from man-made structures. The green areas are volume backscatter from forest canopy. The black linear patterns are the airport runways with low backscatter from their smooth surfaces.

Double bounce scattering events can, however, originate from many natural situations. Among these situations are forest ground-trunk scattering interactions and scattering from abrupt, steep cliff. Man-made structures not aligned in the azimuth direction introduce higher cross-pol returns. This explains the observed strong volume scattering areas in green color which is defined by the strength of $\left|S_{h v}\right|^{2}$.

The agreement between the CP data and the FP data can be tested using a scatter plot which represents the comparison between them as shown in Fig. 10 The three CP- and FPcomponents are seen to be hignly correlated.

\section{Conclusion}

In this study, we have investigated to what extent the CP mode permits to reconstruct the FP information from a single linear transmitted polarization and a reception of two orthogonal polarizations. However, the key property used to estimate the FP information is reflection symmetry, which

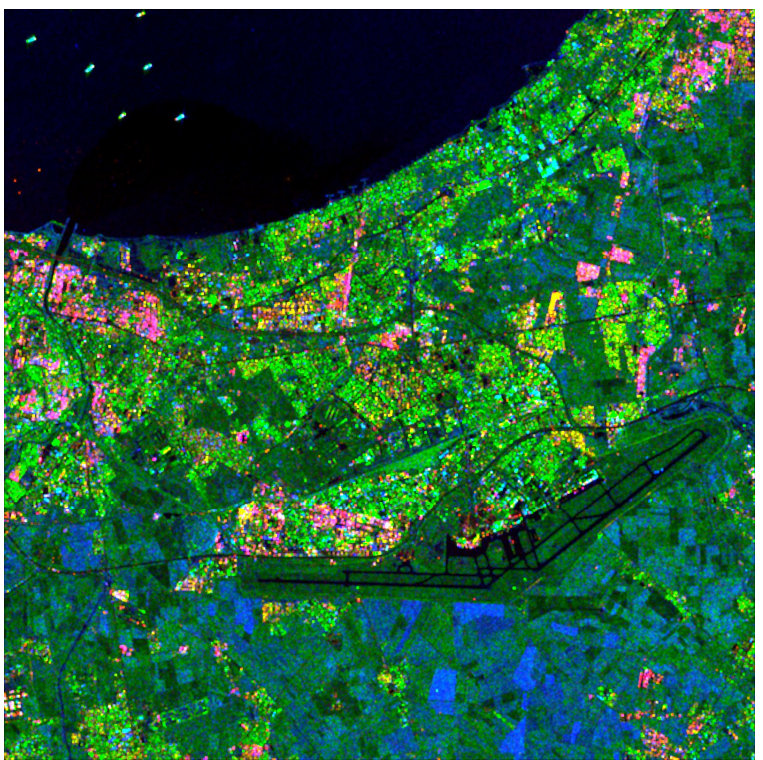

(a)

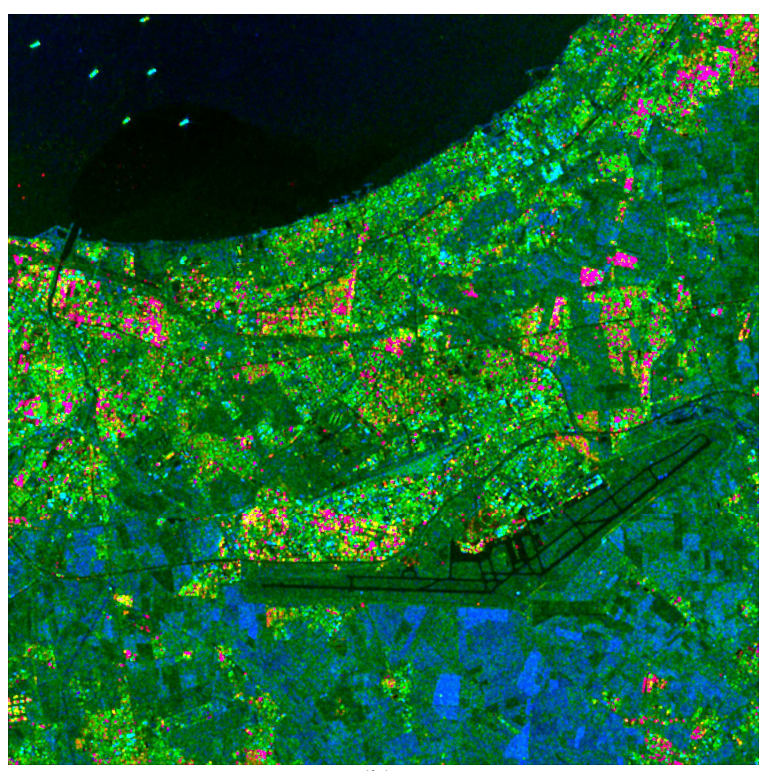

(b)

Figure 9: Freeman-Durden image, the red channel is $f_{d}$, the green channel is $f_{v}$, and the blue channel is $f_{s}$ (a) RGB-full polarimetric image. (b) RGB-compact polarimetric image

reveals a complete decorrelation of copolarized and crosspolarized backscattering coefficients.

We have investigated also the CP mode discriminating capability using polarimetric signatures which provide a complete, easy graphic means to analyze the scattering mechanism information in any mode. In particular, we showed that the $\mathrm{CP}$ signature plots compare well to their corresponding quad-pol.

The Freeman-Durden decomposition based on the symmetry of the geophysical media is derived for the CPand FP-mode. The results indicate that the generated 


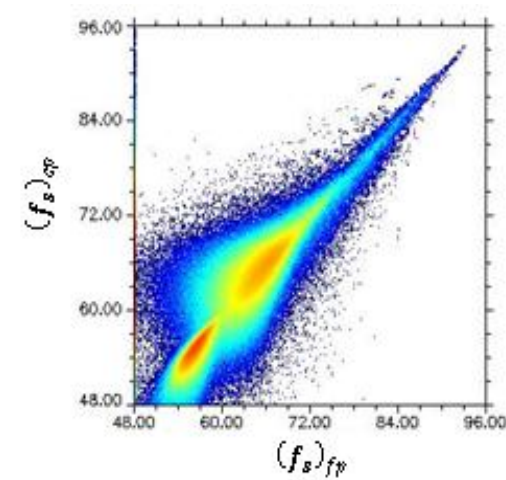

(a)

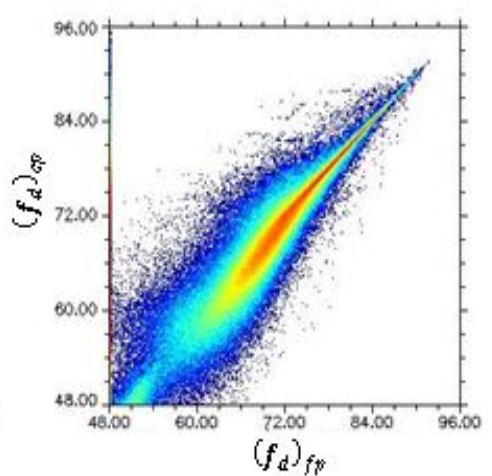

(b)

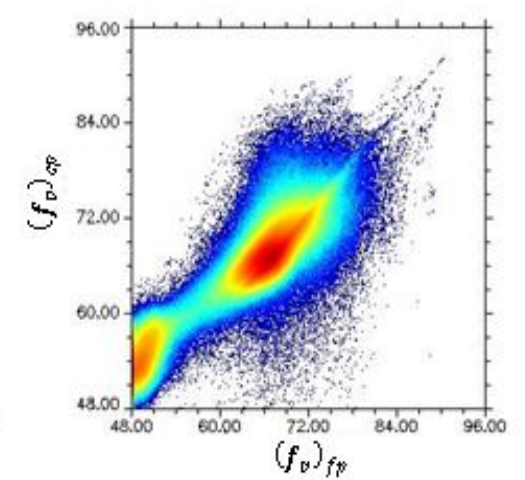

(c)

Figure 10: (a)-(c) scatter plots of the reconstruction performance for odd $\left(\left(\boldsymbol{f}_{s}\right)_{f p}-\left(\boldsymbol{f}_{s}\right)_{c p}\right), \operatorname{double}\left(\left(\boldsymbol{f}_{\boldsymbol{d}}\right)_{f p}-\left(\boldsymbol{f}_{\boldsymbol{d}}\right)_{c p}\right)$ and volume scattering $\left(\left(\boldsymbol{f}_{v}\right)_{f p}-\left(\boldsymbol{f}_{v}\right)_{c p}\right)$ parameters

pseudo quad-pol compares well to the original quad-pol imagery.

As a conclusion, a Compact polarimetry SAR cannot be "as good as" a fully polarimetric system because an FP-SAR measures the $4 \times 4$ covariance scattering matrix of the scene, whereas a CP-SAR has access only to the $2 \times 2$ covariance matrix of the backscattered field. However, in many applications, the results enjoyed from a $\mathrm{CP}$ radar are equivalent to those from an FP radar. Consequently, Compact polarimetry showed promise of being able to reduce the complexity, cost, mass, and data rate of a SAR system while attempting to maintain many capabilities of a fully polarimetric system and it affords more target information than a single-pol system, while not suffering as much from the drawbacks of a quad-pol system.

\section{Acknowledgements}

The authors would like to thank the Canadian Space Agency for kindly providing the polarimetric RADARSAT2 data used in this paper in the frame of the SOAR program under the \#1670 project.

\section{References}

[1] W.-M. Boerner, H. Mott, E. Luneburg, C. Livingstone, B. Brisco, R. J. Brown, and J. S. Paterson, "Polarimetry in radar remote sensing: Basic and applied concepts," in Principles and Applications of Imaging Radar, 3rd ed, vol. 2. F. M. Henderson and A. J. Lewis, Eds. Hoboken, NJ: Wiley, pp. 271-356, 1998.

[2] R. Touzi, W.-M. Boerner, J.-S. Lee, and E. Lueneburg, "A review of polarimetry in the context of synthetic aperture radar: Concepts and information extraction," Can. J. Remote Sens., vol. 30, no. 3, pp. 369-379, 2004.

[3] P. Dubois-Fernandez, J.-C. Souyris, S. Angelliaume, and F. Garestier, "The compact polarimetry alternative for spaceborne SAR at low frequency," IEEE Trans. Geosci. Remote Sens, vol. 46, pp. 3208-3222, 2008.

[4] M-L. Truong-Loï, A. Freeman, P. C. Dubois-Fernandez and E. Pottier, "Estimation of Soil Moisture and Faraday Rotation From Bare Surfaces Using Compact
Polarimetry, " IEEE Trans. Geosci. Remote Sens, vol. 47, no. 11, pp. 3608-3615, 2009.

[5] M. Nord, T. L. Ainsworth, J. -S. Lee, and N. Stacy, "Comparison of Compact Polarimetric Synthetic Aperture Radar Modes, " IEEE Trans. Geosci. Remote Sens., vol. 47, no. 1, pp. 147-188, 2009.

[6] J.-C. Souyris, P. Imbo, R. Fjørtoft, S. Mingot, and J.-S. Lee, "Compact polarimetry based on symmetry properties of geophysical media: The $\pi / 4$ mode," IEEE Trans. Geosci. Remote Sens., vol. 43, no. 3, pp. 634-646, 2005.

[7] K. Raney, "Hybrid polarimetric SAR architecture," IEEE Trans. Geosci.Remote Sens., vol. 45, no. 11, pp. 33973404, 2007.

[8] S. R. Cloude and E. Pottier, "A review of target decomposition theorems in radar polarimetry," IEEE Trans. Geosci. Remote Sens., vol. 34, no. 2, pp. 498-518, 1996.

[9] S. V. Nghiem, S. H. Yueh, R. Kwok, and F. K. Li, "Symmetry properties in polarimetric remote sensing," Radio Sci., vol. 27, no. 5, pp. 693-711, 1992.

[10] M-L. Truong-Loï, "Potentiels d'un système RSO basse fréquence opérant en polarimétrie compacte," Ph.D. dissertation, Univ. Renne1, Renne, France, 2010.

[11] J. J. van Zyl, H. A. Zebker, and C. Elachi, "Imaging radar polarization signatures: Theory and observation," Radio Sci., vol. 22, no. 4, pp. 529-543, 1987.

[12]A. Freeman and S. L. Durden, "A three-component scattering model for polarimetric SAR data," IEEE Trans. Geosci. Remote Sens., vol. 36, no. 3, pp. 963-973, 1998. 